\title{
Liver Support Devices: Bridge to Transplant or Recovery
}

\author{
Nandhini Anamthuruthil Joseph, Lakshmi Krishna Kumar \\ Department of Anesthesia and Critical Care, Amrita Institute of Medical Sciences and Research Centre, Cochin, Kerala, India
}

\section{Abstract}

Liver failure, whether acute or acute on chronic, is a devastating disease with a very high mortality and morbidity. The recent therapeutic advances, especially liver transplant, have given reason for optimism to the ever-rising population affected by this disease. However, scarcity of organs and lack of resources make this an option that only few can afford. The hunt for an artificial device to assist or replace the functions of the liver has been on the rise since the past 40 years. These devices are classified into artificial and bioartificial liver (BAL) assist devices. Artificial liver devices such as molecular adsorbent recirculating system, Prometheus, single-pass albumin dialysis, and selective plasma filtration therapy are mostly aimed at taking over the blood purification systems of the liver. BAL-assisted devices incorporate hepatic cell lines to obtain a more comprehensive coverage of the complex functions of the liver. These include extracorporeal liver assist device, modular extracorporeal liver support, HepatAssist, and Amsterdam Medical Centre-BAL. Development of an ideal liver assist device has been difficult due to the complexity of the functions of the organ. The initial studies on these devices are promising but inconclusive. Therapeutic plasma exchange seems to have a very favorable profile in the treatment of these patients and has been successfully used in a large number of patients. To arrive at a more definitive conclusion of the usefulness of these devices in the management of liver failure, large randomized multicentric studies with more objective end points need to be carried out. A literature review was performed using PubMed and library searches to collect the recent studies in this regard. This review aims to provide a myopic view of the advances that have been made in the development and usefulness of these liver assist devices.

Keywords: Artificial liver, bioartificial liver, liver assist devices, liver failure

\section{INTRODUCTION}

The high mortality following liver failure, whether acute or acute on chronic, has not changed significantly over the years. While some patients may tide over the crisis period, majority succumb to liver failure waiting for a transplant. Liver transplant has definitely revolutionized the management of these patients. The 1-year survival following liver transplant is $95 \%$. However, this still remains a luxury that only few can afford and many die waiting for a donor. According to the Global Observatory on Donation and Transplantation, approximately 20,200 liver transplants are being conducted every year. In India alone, 1296 transplants are being conducted annually. This is much lower than our annual requirement of 50,000 liver transplants. In majority of the developed countries, $70 \%-95 \%$ of the liver transplants are cadaveric donor transplants. On the other hand, the liver transplant program in India is driven by live donors with $94.5 \%$ of our transplants being from live donors. ${ }^{[1]}$ The cultural, social, and economical factors may explain the huge lacunae in our supply-demand ratio. The liver is one of the

\begin{tabular}{|l|l|}
\hline \multicolumn{2}{|c|}{ Access this article online } \\
\hline Quick Response Code: & Website: \\
& www.ijrconline.org \\
\cline { 2 - 2 } & \\
\hline
\end{tabular}

largest organs in our body with important synthetic, excretory, and immunogenic functions. The liver is the only organ in our body that has the capacity to regenerate, and hepatocyte destruction remains clinically silent till $70 \%$ of the tissue has been damaged. ${ }^{[2]}$ In an attempt to support patients while the liver recovers or waiting for a transplant, various liver support devices have been designed. The ideal liver support device which would take over all the functions of the liver is still a dream that needs to be materialized. In this article, we review the recent literature using PubMed and library searches on liver support devices and their outcome on patient mortality and morbidity.

\section{Liver: A Vital Organ}

The liver is the largest organ in our body and accounts for approximately $2 \%$ of the total mass of healthy adults. The

Address for correspondence: Dr. Nandhini Anamthuruthil Joseph, Department of Anesthesia and Critical Care, Amrita Institute of Medical Sciences and Research Centre, Cochin, Kerala - 682 024, India. E-mail: nandhinijoseph@gmail.com

This is an open access article distributed under the terms of the Creative Commons Attribution-NonCommercial-ShareAlike 3.0 License, which allows others to remix, tweak, and build upon the work non-commercially, as long as the author is credited and the new creations are licensed under the identical terms.

For reprints contact: reprints@medknow.com

How to cite this article: Joseph NA, Kumar LK. Liver support devices: Bridge to transplant or recovery. Indian J Respir Care 2017;6:807-12. 
complexity of the functions carried out by the liver makes it indispensable for the normal functioning of our body. ${ }^{[3]}$

The functions carried out by the liver include: ${ }^{[3]}$

- $\quad$ Protein, fat, and carbohydrate metabolism

- Synthesis and activation of coagulants and procoagulants

- $\quad$ Erythropoiesis and erythrocytosis

- Metabolism and synthesis of hormones and hormone-binding proteins

- Modulators of immune and inflammatory responses

- Drug metabolism and excretion

- As a storage organ.

\section{Liver FalluRe}

Liver is a remarkable organ with the ability to compensate even when up to $70 \%$ of liver tissue is destroyed and with the ability to regenerate. Liver failure is a life-threatening disorder when large parts of the liver become damaged and the liver is no longer able to function. This can be acute or chronic.

Chronic liver failure occurs as a consequence of the gradual destruction of hepatocytes, and the functional capacity of the liver can no longer maintain the normal physiological conditions over a period of months. The most common causes are alcohol-induced, viral hepatitis, hepatocellular carcinoma, and autoimmune diseases. This can also be an acute-on-chronic injury which may occur during viral/alcoholic hepatitis or when there are increased metabolic demands, for example, infection or gastrointestinal hemorrhage. In chronic liver failure, ascites, encephalopathy, hepatic dysfunction, and portal hypertension are observed. ${ }^{[4]}$

Acute liver failure occurs when there is an onset of encephalopathy within 8-12 weeks of onset of jaundice in the absence of any evidence of preexisting liver disease. Depending on the time interval between the onset of symptoms and onset of encephalopathy, it is further classified into hyperacute, acute, and subacute according to O'Grady system of classification. The clinical presentation includes encephalopathy, coagulation abnormalities, and hepatic dysfunction. Ascites and portal hypertension are rarely seen. The common causes are viral hepatitis, drug-induced, and ischemic or hypoxic hepatocellular injury. This has a very high mortality rate. ${ }^{[5]}$

In either case, the only treatment available is supportive till the patient improves or undergoes liver transplantation. In the absence of this, patients with liver failure have a very high mortality rate. ${ }^{[6]}$

\section{Liver support devices}

In an attempt to tide over the crisis period during liver failure till the liver regenerates or a transplant organ becomes available, a number of liver assist devices have been designed. Unlike kidney, heart, or lung, the complexity of functions of the liver has made it difficult to materialize such a device.

An ideal "artificial liver" would perform three key functions of the liver: detoxification, biosynthesis, and regulation. Thus, it should be able to lower the blood levels of toxic substances and should provide all liver functions that have been lost. It would also facilitate the regeneration of native hepatocytes and hence recovery. ${ }^{[7,8]}$

This ideal device would assist patients with borderline functional liver mass till a donor becomes ready or the liver regenerates. It would also help patients during periods of functional recovery as after a transplant or after extended liver resections after trauma or cancer. ${ }^{[7]}$

To understand the various liver assist devices better, it is imperative to have a better understanding of two terms: blood detoxification and blood purification.

In the beginning, most liver assist devices were designed solely concentrated on blood detoxification. It was assumed then that hepatic encephalopathy was caused as a result of the accumulation of toxins which were small dialysable molecules. These initial detoxification devices were based on sorbents (charcoal and resin) that treat the whole blood or plasma or dialysate. ${ }^{[9-11]}$ Further studies demonstrated that the clinical features of hepatic failure can be attributed to much more complicated biochemical abnormalities. ${ }^{[12]}$ These substances that accumulate in the blood cause neurological abnormalities, aggravate injury to the liver and other organs, inhibit hepatocyte functions, and suppress hepatic regeneration. They include small molecular weight toxins, mediators of inflammation, vasoactive substances, endotoxins, cell growth inhibitors, and many unknown substances. A large number of the toxins are bound to proteins or exist as multimers and cannot be removed by the traditional sorption techniques. This could explain the failure of the initial liver assist devices that were designed based on blood detoxification techniques. The concept of blood purification has given a better understanding for the development of new liver assist devices. These devices aim at removing all known substances that accumulate in the blood during hepatic failure and not just the toxins. ${ }^{[6]}$ Recent years have seen a surge in the development of these devices. The utility and efficacy of these new therapeutic modalities are currently being tested in the clinical settings.

\section{Therapeutic plasma exchange (Plasmapheresis)}

The removal of patient's plasma and replacing it with plasma from a donor using an extracorporeal device refers to therapeutic plasma exchange (TPE). This has been found to be a very effective method of attaining blood purification in liver failure patients. This increases hepatic blood flow and decreases blood ammonia levels. The TPE in addition also has the advantage of providing deficient clotting factors and albumin in these patients. ${ }^{[13-17]}$ However, this achievement of blood purification is also accompanied with a lowering of the blood hepatocyte growth factor (HGF) levels and is also not effective in removing the intracellular toxins that accumulate. These plasma transfusions also carry the risk of hypocalcemia, metabolic acidosis, pulmonary and cerebral complications. ${ }^{[6]}$ Nevertheless, TPE continues to be one of the most frequently 
used methods of liver support for patients with acute hepatic failure.

\section{Classification of liver assist devices}

The liver assist devices can be classified into two major groups: artificial liver support devices and bioartificial liver support devices [Table 1]. ${ }^{[18]}$

Artificial liver support devices are noncell-based devices that mainly carry out the function of blood detoxification and blood purification. Human blood toxic substances can be classified into water soluble (ammonia, creatinine, interleukins (ILs), etc.) or protein bound (bilirubin, benzodiazepines, nitric oxide, etc). Conventional techniques such as hemodialysis or hemofiltration remove only the water-soluble toxins. The protein-bound toxins can be removed only by addition of albumin to the dialysate or the use of large-pore filters. ${ }^{[8]}$

Bioartificial liver support devices are cell-based liver support devices. They have a cellular component such as primary hepatocytes or hepatic cell lines. In majority of these devices, the hepatic cell lines are derived from porcine hepatocytes or from tumor cell line or harvested from organs that are deemed unsuitable for transplant. The former two cell lines raise safety concerns regarding infection and malignancy transmission. ${ }^{[7]}$ Human hepatocytes harvested from organs are in scarcity and stem cell research holds a promising future in this regard. ${ }^{[18]}$ Some are of the opinion that porcine hepatocytes are the safest candidates for use until a liver cell line that is safe, proliferates and maintains differentiated functions becomes available. ${ }^{[6]}$ Although infection of human cell with pig endogenous retrovirus (PERV) has been demonstrated in culture systems, no PERV transmission has been documented in humans transplanted with porcine cells or who had contact with pig cells or tissues so far. ${ }^{[19,20]}$ The cellular components in these devices are intended to replace the important liver functions such as synthesis, detoxification, biotransformation, and excretion. Recently, an immortalized human fetal cell line (CBAL1111) has been developed which may open new avenues in the development of biological devices. $^{[21]}$

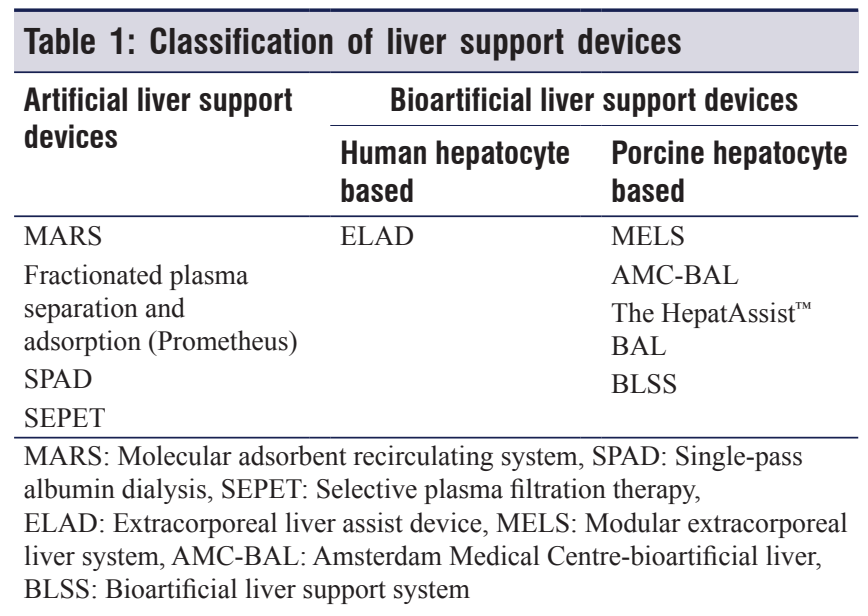

\section{Artificial Liver Support Devices} Molecular adsorbent recirculating/recycling system

Molecular adsorbent recirculating system (MARS) was developed in the 1990s by Stange et al. in Sweden. They combined conventional dialysis against an albumin dialysate followed by a conventional dialysis procedure to remove the toxins from the dialysate.

MARS system consists of two circuits: The blood circuit and the secondary circuit. The blood circuit passes the patient's blood over an albumin impermeable membrane through a high-flux dialyzer. The opposing side of the membrane contains $600 \mathrm{ml}$ of $20 \%$ albumin in the secondary circuit. The toxins will diffuse across the membrane and bind to the albumin on the other side. The albumin in the secondary circuit is then cleared of toxins by anion exchange resin and activated charcoal columns. MARS has been found to be effective in removing free bilirubin and bilirubin bound to albumin by noncovalent forces. ${ }^{[22]}$

MARS has been found to reduce bilirubin levels, encephalopathy, pruritus, and serum copper levels in Wilson's disease. Improvement in renal function, cerebral blood flow, and varied effects on intracranial pressure (ICP) have also been reported. ${ }^{[23-25]}$ At the same time, it has also been noticed that MARS might worsen coagulopathy, hypoglycemia, and alter the pharmacokinetics of drugs. ${ }^{[26]}$

The overall effect of MARS on mortality seems inconclusive. Though initial studies with MARS showed promising results, a meta-analysis of four randomized trials failed to show any survival benefits. In fact, the short-term mortality rates for patients treated with MARS but without transplantation ranged from $78 \%$ to $100 \% .{ }^{[18]}$ MARS may be used to stabilize patients prior to transplantation and for allograft dysfunction after transplantation till the liver recovers. It may not improve survival without transplantation. ${ }^{[23-25]}$

\section{Fractionated plasma separation and adsorption (Prometheus)}

The Prometheus system developed in Germany purified blood without the use of exogenous albumin. In this system, the blood is passed over the AlbuFlow $250 \mathrm{kDa}$ membrane which is permeable to albumin. The albumin-bound toxins pass through the albumin permeable membrane and the filtrate is passed through a column of neutral resin and anion exchange resin and returned to the patient. This removes the toxins from the albumin and is returned to the patient. The water-soluble, low molecular weight toxins are removed downstream with a high-flux hemodialysis. It is postulated that patients treated with Prometheus would be detoxified much more effectively than those treated with MARS. However, the clinical experience with this system is limited and no definite conclusions can be made as of now. ${ }^{[27,28]}$ In one study comparing MARS versus Prometheus in patients with alcoholic hepatitis or alcoholic cirrhosis, it was found that mean arterial pressure and systemic vascular resistance improved better with MARS in comparison to Prometheus. ${ }^{[29]}$ However, bleeding 
complications with Prometheus are rare and there might even be the need to use anticoagulation during the procedure. ${ }^{[28]}$

\section{Single-pass albumin dialysis}

Single-pass albumin dialysis (SPAD) is a very simple and inexpensive technique of blood purification where the need for additional circuits is discarded. It is a simple venovenous hemodialysis where the dialysate solution contains low concentration albumin (4.4\%). The albumin toxin complexes are then discarded and not regenerated. ${ }^{[18]} \mathrm{A}$ single randomized controlled study has shown that MARS and SPAD were equally effective in reducing plasma bilirubin levels. However, only MARS affected other paraclinical parameters such as serum bile acids, albumin-binding capacity, creatinine and urea levels. ${ }^{[30]}$ Preliminary clinical experience shows that SPAD has a promising future with its simplicity and low cost. ${ }^{[31]}$

\section{Selective plasma filtration therapy}

Selective plasma filtration therapy (SEPET) is a concept that evolved in California when Rozga and colleagues studied the humoral, cellular, and molecular mechanisms that regulate recovery of diseased liver. They observed that, after loss of massive hepatic parenchyma, the blood levels of both positive (HGF) and negative transforming growth factor beta $1\left(\mathrm{TGF}^{\beta} 1\right)$ regulators of hepatocyte proliferation and pro-inflammatory cytokines (IL-6, tumor necrosis factor-alpha) increase substantially. They hypothesized that removal of pro-inflammatory cytokines and $\mathrm{TGF}^{\beta}$ with preservation of high blood HGF levels may be an effective liver support therapy and promotes liver regeneration. The most important contribution was that all the currently known mediators of inflammation and inhibitors of hepatocyte regeneration have a molecular weight $<100 \mathrm{kDa}$. SEPET is a blood filtration device that removes the plasma fraction that contains these elements while retaining important blood components with molecular weight $>100 \mathrm{kDa}$ which include HGF, blood clotting factors, and complement system proteins. The removed plasma fraction is replaced with electrolyte solution, human albumin, and fresh frozen plasma ${ }^{[6]}$ Preclinical studies have shown it to be effective in extending survival time and arresting brain swelling. ${ }^{[32]}$ In an uncontrolled study, 11 of 14 patients showed improvement in hepatic encephalopathy ${ }^{[6]}$ Further studies will be needed to arrive at some definitive conclusion regarding this novel device.

\section{Bioartificial Liver Support Devices}

The bioartificial liver support devices as mentioned earlier are cell-based support devices. The last decade has seen a major surge in the development of these devices. Majority of these devices are porcine hepatocyte based.

\section{Extracorporeal liver assist device}

Extracorporeal liver assist device (ELAD) is based on hepatoblastoma $\mathrm{C} 3 \mathrm{~A}$ cell line. This device was initially evaluated in King's College Hospital in London. The original device was assessed in 24 patients with acute liver failure. The device consisted of exposing the patient's whole blood for duration of about $3-168 \mathrm{~h}$ to the hepatocytes. The functioning cell mass was estimated to be about $80-90 \mathrm{~g}$ based on the rate of oxygen consumption of the device. The study, however, proved inconclusive in terms of survival rate. ${ }^{[33]}$

Subsequently, modifications were done in the device to improve its efficiency and properties. These include increasing the functional cell mass to $300-400 \mathrm{~g}$ in adults, introducing oxygenation and nutritional components in the circuit to improve cell viability, whole blood exposure was replaced with ultra-filtrate exposure, and increasing pore size of the membrane to facilitate free movement of molecules in the device. This improved device was then evaluated in 25 patients who fulfilled criteria for liver transplant. The ELAD-treated and control patients had a similar 30-day survival rate. However, among the 19 patients who were listed for transplant, the ELAD-treated patients had a much higher 30-day survival rate of $81 \%$ in comparison to $56 \%$ in the control group. ${ }^{[34]}$ This led to the idea of liver assist devices as a bridge to transplantation rather than a bridge to recovery. Further studies in other countries are planned to be conducted to assess the clinical relevance of these findings.

\section{HepatAssist}

HepatAssist ${ }^{\mathrm{TM}}$ by Arbios was the first bioartificial liver device that was Food and Drug Administration approved for Phase I/II/III clinical trials. It is also the first bioartificial liver where a hybrid concept of combining hepatocyte bioreactor with a column filled with adsorbent was used.

In this bioartificial liver, the patient's blood is first separated into plasma and cellular components. The plasma is then passed through a high-flow plasma circulation loop. The plasma is passed successively through a charcoal filter, oxygenator, heater, and a hollow fiber bioreactor containing 7 billion cryopreserved hepatocytes. The plasma that has been purified and processed is then united with the cellular components and returned to the patient. ${ }^{[6]}$

In the Phase I study, patients received standard medical care along with treatment with HepatAssist ${ }^{\mathrm{TM}}$. Thirty-two patients were enrolled in the study and it was tolerated well with no technical problems. Majority of the patients had fulminant hepatic failure and three patients had primary nonfunction of the allograft postliver transplantation. The survival rate was $84 \%$ with or without transplantation. The patients experienced remarkable neurological improvement with significant reduction in ICP and improvement in cerebral perfusion pressure. There was also a decrease in ammonia, transaminase, and bilirubin levels. ${ }^{[35]}$

In Phase II/III clinical trials, 171 patients ( 86 controls and 85 treated) were enrolled in 11 US and 9 European medical centers. It was conducted to study the efficacy of this device in patients with acute liver failure. Patients with Stage III or IV hepatic encephalopathy or with primary nonfunction of the transplanted liver were considered. Patients were randomized into two groups. The control group received standard of care treatment. 
Joseph and Kumar: Liver support devices

The treated group received standard of care treatment along with daily treatment with HepatAssist ${ }^{\mathrm{TM}}$ of $7 \mathrm{~h}$ duration. This would continue till neurological recovery or transplantation or the occurrence of an adverse event that prevented further treatment. Though the trials verified a positive safety profile, it failed to improve 30-day survival in the study population. On adjustment for impact of liver transplantation and median time to transplant favoring controls, the treated groups had a statistically significant survival advantage over the controls. Subgroup analysis suggested that HepatAssist ${ }^{\mathrm{TM}}$ therapy may provide a beneficial survival rate in patients with drug and chemical toxicity-induced hepatic failure. ${ }^{[36]}$ An improved version of HepatAssist ${ }^{\mathrm{TM}}$ with 15-20 billion porcine hepatocytes will be tested in Phase III clinical trials according to Arbios Systems, Inc. ${ }^{[6]}$

\section{Modular extracorporeal liver support}

This BAL support system developed in Germany is based on hollow fibers containing fresh porcine hepatocytes. The initial study conducted in a small population of eight patients with acute liver failure has shown that it may be useful as a bridge to transplant. However, its high cost and complex design preclude its use in a more widespread manner. ${ }^{[6]}$

\section{Bioartificial liver support system}

This is a system developed in Minnesota, USA, and consists of porcine hepatocytes in a single hollow fiber cartridge. Clinical trials are still underway to assess its safety and utility. ${ }^{[8]}$

\section{Amsterdam Medical Centre-Bioartificial Liver}

This system also utilizes porcine hepatocytes bound to a spiral-shaped polyester fabric with an integrated hollow fiber. In contrary to other bioartificial liver (BAL) support devices, this device does not separate the patient's plasma from the cells by a membrane. The system is incorporated with capillaries for oxygenation and viability. ${ }^{[37]}$ Even though the preliminary studies are promising, much larger trials are required for definitive evidence. ${ }^{[6]}$

An in vitro comparative study between modular extracorporeal liver support (MELS) and Amsterdam Medical Centre (AMC)-BAL has shown a comparable functional capacity over a period of 7 days. However, there was difference in three parameters that were studied. It was observed that lidocaine and ammonia elimination was significantly higher in AMC-BAL as compared to MELS. The LDH release was found to be significantly lower in the MELS group over the period of 7 days. ${ }^{[38]}$

Apart from the above-mentioned devices, a number of other BAL support devices have been developed around the world. These include but are not limited to the TECA-Hybrid Artificial Liver Support System (Beijing, China), the Hybrid-BAL (Nanjing, China), the Bioartificial Hepatic Support (Udine, Italy), and the Radial Flow Bioreactor (Ferrara, Italy). ${ }^{[18]}$

\section{Future of Liver Assist Devices}

The recent surge in the development of liver assist devices in the recent years has led to a lot of investment in this field.
However, till date, there has been no conclusive evidence demonstrating a clear-cut advantage of these devices over the standard of care treatment. A 2004 Cochrane systematic review came to the conclusion that liver support devices improved hepatic encephalopathy and did not reduce mortality in all cases of liver failure, especially acute liver failure. An optimistic finding was the $33 \%$ reduction in mortality in acute-on-chronic liver failure. However, this review has to be criticized in its lack of strength of evidence. They analyzed only 12 randomized controlled trials and most of them had methodological limitations. ${ }^{[39]}$ This further reiterates the need for larger randomized controlled trials in a more homogeneous population with specific end points of these devices to assess their real benefits. The difficulty in performing such studies, especially as majority of the patients will be diverted to liver transplantation, cannot be underestimated.

Realizing this need, a number of multicentric randomized controlled trials are ongoing or being planned with survival and/or improvement in hepatic encephalopathy as their primary outcome. They include and are not limited to four trials on MARS; the European Helios trial on Prometheus, a European trial on Plasmapheresis, a trial on SEPET, and a Chinese trial on ELAD. ${ }^{[18]}$

\section{ConcLusion}

The requirement of the ideal liver assist device is very high, and at the same time, the development of this device still remains vague. A lot of developments have been made in the recent years, but more definitive evidence will be required before it can be incorporated into standard of care treatment in liver failure. Till then, transplantation and supportive therapy remains the only solace for these patients with a high mortality otherwise. The enthusiasm in this field is warranted with the initial promising results. The change in paradigm from bridge to recovery to bridge to transplant with these devices seems more realistic in the current scenario.

\section{Financial support and sponsorship}

Nil.

\section{Conflicts of interest}

There are no conflicts of interest.

\section{ReFERENCES}

1. Data (Charts and Tables) - Global Observatory on Donation and Transplantation. Available from: http://www.transplant-observatory.org/ data-charts-and-tables. [Last retrieved on 2017 Apr 27].

2. Mushlin PS, Gelman S. Hepatic physiology and pathophysiology. In: Miller RD, editor. Miller's Anesthesia. $7^{\text {th }}$ ed. Philadelphia: Churchill Livingstone; 2010. p. 428-31.

3. Mushlin PS, Gelman S. Hepatic physiology and pathophysiology. In: Miller RD, editor. Miller's Anesthesia. $7^{\text {th }}$ ed. Philadelphia: Churchill Livingstone; 2010. p. 412-24.

4. Chapman RW, Collier JD, Hayes PC. Liver and biliary tract disease. In: Boon NA, Colledge NR, Walker BR, Hunter JA, editors. Davidson's Principles and Practices of Medicine. 20 $0^{\text {th }}$ ed. Philadelphia: Churchill Livingstone; 2006. p. 954-5.

5. Bernal W, Wendon J. Acute liver failure. N Engl J Med 2014;370:1170-1. 
Joseph and Kumar: Liver support devices

6. Rozga J. Liver support technology - An update. Xenotransplantation 2006;13:380-9.

7. O'Grady J. Personal view: Current role of artificial liver support devices. Aliment Pharmacol Ther 2006;23:1549-57.

8. Puri P, Anand AC. Liver support devices. Med Update 2012;22:489-93.

9. Slack AJ, Auzinger G, Willars C, Dew T, Musto R, Corsilli D, et al. Ammonia clearance with haemofiltration in adults with liver disease. Liver Int 2014;34:42-8.

10. O’Grady JG, Gimson AE, O'Brien CJ, Pucknell A, Hughes RD, Williams R. Controlled trials of charcoal hemoperfusion and prognostic factors in fulminant hepatic failure. Gastroenterology 1988;94(5 Pt 1):1186-92.

11. Gimson AE, Braude S, Mellon PJ, Canalese J, Williams R. Earlier charcoal haemoperfusion in fulminant hepatic failure. Lancet 1982;2:681-3

12. Coltart I, Tranah TH, Shawcross DL. Inflammation and hepatic encephalopathy. Arch Biochem Biophys 2013;536:189-96.

13. Liu CT, Chen TH, Cheng CY. Successful treatment of drug-induced acute liver failure with high-volume plasma exchange. J Clin Apher 2013;28:430-4.

14. Larsen FS, Schmidt LE, Bernsmeier C, Rasmussen A, Isoniemi H, Patel VC, et al. High-volume plasma exchange in patients with acute liver failure: An open randomised controlled trial. J Hepatol 2016;64:69-78.

15. Karvellas CJ, Subramanian RM. Current evidence for extracorporeal liver support systems in acute liver failure and acute-on-chronic liver failure. Crit Care Clin 2016;32:439-51.

16. Iwai $\mathrm{H}$, Nagaki $\mathrm{M}$, Naito $\mathrm{T}$, Ishiki $\mathrm{Y}$, Murakami $\mathrm{N}$, Sugihara J, et al. Removal of endotoxin and cytokines by plasma exchange in patients with acute hepatic failure. Crit Care Med 1998;26:873-6.

17. Akdogan M, Camci C, Gurakar A, Gilcher R, Alamian S, Wright H, et al. The effect of total plasma exchange on fulminant hepatic failure. J Clin Apher 2006;21:96-9.

18. Phua J, Lee KH. Liver support devices. Curr Opin Crit Care 2008;14:208-15.

19. Patience $C$, Takeuchi $Y$, Weiss RA. Infection of human cells by an endogenous retrovirus of pigs. Nat Med 1997;3:282-6.

20. Pitkin Z, Mullon C. Evidence of absence of porcine endogenous retrovirus (PERV) infection in patients treated with a bioartificial liver support system. Artif Organs 1999;23:829.

21. Poyck PP, van Wijk AC, van der Hoeven TV, de Waart DR, Chamuleau RA, van Gulik TM, et al. Evaluation of a new immortalized human fetal liver cell line (cBAL111) for application in bioartificial liver. J Hepatol 2008;48:266-75.

22. Stange J, Mitzner SR, Risler T, Erley CM, Lauchart W, Goehl H, et al. Molecular adsorbent recycling system (MARS): Clinical results of a new membrane-based blood purification system for bioartificial liver support. Artif Organs 1999;23:319-30.

23. Bachli EB, Schuepbach RA, Maggiorini M, Stocker R, Müllhaupt B, Renner EL. Artificial liver support with the molecular adsorbent recirculating system: Activation of coagulation and bleeding complications. Liver Int 2007;27:475-84.

24. Stange J, Hassanein TI, Mehta R, Mitzner SR, Bartlett RH. The molecular adsorbents recycling system as a liver support system based on albumin dialysis: A summary of preclinical investigations, prospective, randomized, controlled clinical trial, and clinical experience from 19 centers. Artif Organs 2002;26:103-10.

25. Mitzner SR, Stange J, Klammt S, Koball S, Hickstein H, Reisinger EC. Albumin dialysis MARS: Knowledge from 10 years of clinical investigation. ASAIO J 2009;55:498-502.

26. Bañares R, Nevens F, Larsen FS, Jalan R, Albillos A, Dollinger M, et al. Extracorporeal albumin dialysis with the molecular adsorbent recirculating system in acute-on-chronic liver failure: The RELIEF trial. Hepatology 2013;57:1153-62.

27. Rifai K, Ernst T, Kretschmer U, Bahr MJ, Schneider A, Hafer C, et al. Prometheus - A new extracorporeal system for the treatment of liver failure. J Hepatol 2003;39:984-90.

28. Rifai K, Manns MP. Review article: Clinical experience with Prometheus. Ther Apher Dial 2006;10:132-7.

29. Krisper P, Stauber RE. Technological insight: Artificial extracorporeal liver support - How does Prometheus compare with MARS? Nat Rev Nephrol 2007;3:267-76.

30. Sponholz C, Matthes K, Rupp D, Backaus W, Klammt S, Karailieva D, et al. Molecular adsorbent recirculating system and single-pass albumin dialysis in liver failure - A prospective, randomised crossover study. Crit Care 2016;20:2.

31. Boonsrirat U, Tiranathanagul K, Srisawat N, Susantitaphong P, Komolmit $\mathrm{P}$, Praditpornsilpa $\mathrm{K}$, et al. Effective bilirubin reduction by single-pass albumin dialysis in liver failure. Artif Organs 2009;33:648-53

32. Rozga J, Umehara Y, Trofimenko A, Sadahiro T, Demetriou AA. A novel plasma filtration therapy for hepatic failure: Preclinical studies. Ther Apher Dial 2006;10:138-44.

33. Ellis AJ, Hughes RD, Wendon JA, Dunne J, Langley PG, Kelly JH, et al. Pilot-controlled trial of the extracorporeal liver assist device in acute liver failure. Hepatology 1996;24:1446-51.

34. Millis JM, Cronin DC, Johnson R, Conjeevaram H, Conlin C, Trevino S, et al. Initial experience with the modified extracorporeal liver-assist device for patients with fulminant hepatic failure: System modifications and clinical impact. Transplantation 2002;74:1735-46.

35. Watanabe FD, Mullon CJ, Hewitt WR, Arkadopoulos N, Kahaku E, Eguchi S, et al. Clinical experience with a bioartificial liver in the treatment of severe liver failure. A phase I clinical trial. Ann Surg 1997;225:484-91.

36. Demetriou AA, Brown RS Jr., Busuttil RW, Fair J, McGuire BM, Rosenthal $\mathrm{P}$, et al. Prospective, randomized, multicenter, controlled trial of a bioartificial liver in treating acute liver failure. Ann Surg 2004;239:660-7.

37. Pless G. Artificial and bioartificial liver support. Organogenesis 2007;3:20-4.

38. Poyck PP, Pless G, Hoekstra R, Roth S, Van Wijk AC, Schwartländer R, et al. In vitro comparison of two bioartificial liver support systems: MELS CellModule and AMC-BAL. Int J Artif Organs 2007;30:183-91.

39. Liu JP, Gluud LL, Als-Nielsen B, Gluud C. Artificial and bioartificial support systems for liver failure. Cochrane Database Syst Rev 2004;(1):CD003628 